Pacific Journal of Mathematics

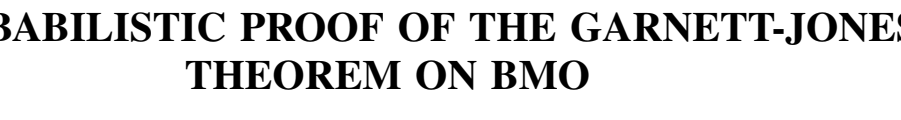




\title{
A PROBABILISTIC PROOF OF THE GARNETT-JONES THEOREM ON BMO
}

\author{
N. Th. VAROPOULOS
}

I give a probabilistic proof (via Brownian motion) of the real variable Garnett-Jones Theorem, which states that there exists some constant $C_{n}$, depending only on the dimension $n$, such that for all $f \in \mathrm{BMO}\left(\boldsymbol{R}^{n}\right)$ in the John-Nirenberg class 1 we have distance $\left(f, L^{\infty}\right) \leq C_{n}$ (the distance being measured in the BMO norm).

o. Introduction.

0.1. Statement of the theorems. Let $f \in L_{\mathrm{loc}}^{1}\left(\boldsymbol{R}^{n}\right)(n \geqq 1)$. We then say that $f \in \operatorname{BMO}\left(\boldsymbol{R}^{n}\right)$ if:

$$
\sup _{I} \frac{1}{|I|} \int_{I}\left|f-f_{I}\right| d x=\|f\|_{\text {вмо }}<+\infty
$$

$I$ in the above expression runs through all cubes with sides parallel to the axes, $|I|$ denotes the Euclidean measure of $I$ and

$$
f_{I}=\frac{1}{|I|} \int_{I} f d x \text {. }
$$

It is well known that if $f \in$ BMO then there exists some $\alpha>0$ such that:

$$
\sup _{I} \frac{1}{|I|} \int_{I} e^{\alpha\left|f-f_{I}\right|} d x<+\infty
$$

where $I$ runs through the same collection as above. Let us denote by $\alpha_{0}=\alpha_{0}(f)>0$ the supremum of all $\alpha$ 's for which (0.1.2) holds. $\alpha_{0}$ can then be used to estimate the distance of $f$ from $L^{\infty}$ in BMO. More precisely we have the following theorem which is due to John Garnett and Peter Jones [4].

THEOREM (G.J.). There exist two constants $C_{1}, C_{2}>0$ that only depend on the dimension $n$ such that for all $f \in \operatorname{BMO}\left(\boldsymbol{R}^{n}\right)$ we have

$$
\frac{C_{2}}{\alpha_{0}} \leqq \inf _{\psi \in L^{\infty}}\|f-\psi\|_{\text {вмо }} \leqq \frac{C_{1}}{\alpha_{0}} .
$$

In this note I propose to give a probabilistic proof of the above theorem that goes via Brownian motion. To state the relevant theorem from probability theory I shall need to introduce some notation. 
Let $(\Omega, \mathscr{F}, \boldsymbol{P})$ be a probability space; for any subfield $\mathscr{G} \subset \mathscr{F}$ I shall denote by $\boldsymbol{E}(f / / \mathscr{G})$ the conditional expectation of $f \in L^{1}(\Omega)$ with respect to the subfield $\mathscr{G}$. Let now $\left(\mathscr{F}_{t} \subset \mathscr{F} ; t \geqq 0\right)$ be an increasing family of subfields $\left(t_{1}<t_{2} \Rightarrow \mathscr{F}_{t_{1}} \subset \mathscr{F}_{t_{2}}\right)$ such that $\cup \mathscr{F}_{t}=\mathscr{F}$, and for any $f \in L^{1}(\Omega)$ let us denote

$$
f_{t}=E\left(f / / \mathscr{F}_{t}\right) \quad t \geqq 0 .
$$

I shall make the following hypothesis on $\left(\Omega ; \mathscr{F} ; \mathscr{F}_{t}, t \geqq 0 ; \boldsymbol{P}\right)$ which will be assumed to be verified by all the spaces that we shall consider in this paper.

Hypothesis $(\mathrm{H})$. For all $f \in L^{1}(\Omega)$ there exists a family of functions $\left(\widetilde{f}_{t} \in L^{1}(\Omega), t \geqq 0\right)$ such that:

$$
\widetilde{f}_{t}=f_{t} \text { a.s. on } \Omega
$$

for all $t \geqq 0$, and such that for almost all $\omega \in \Omega$ the function

$$
t \longrightarrow \widetilde{f}_{t}(\omega),
$$

is a continuous function of $t \geqq 0$.

The above hypothesis requires that the $L^{1}$-martingales with respect to the subfields $\left(\mathscr{F}_{t}, t \geqq 0\right.$ ) should all have "continuous paths". This hypothesis, as we shall see, is widely verified in nature.

Let now $\Omega$ be as above and let $f \in L^{1}(\Omega)$. We then say that $f \in \operatorname{BMO}(\Omega)$ if there exists a constant $K>0$ such that

$$
\boldsymbol{E}\left[\left|f-f_{t}\right| / / \mathscr{F}_{t}\right] \leqq K, \quad t \geqq 0 .
$$

The Garnett-Jones theorem then has the following probabilistic analogue.

Theorem (G.J. Prob.). Let $\Omega$ be a probability space as above. Then there exist two constants $C_{1}, C_{2}>0$ such that for all $f \in \operatorname{BMO}(\Omega)$ we have:

$$
\frac{C_{2}}{\alpha_{0}} \leqq \inf _{\psi \in L^{\infty}}\|f-\psi\| \leqq \frac{C_{1}}{\alpha_{0}}
$$

where $\alpha_{0}$ is the supremum of all $\alpha \geqq 0$ that satisfy

$$
\sup _{t}\left\|\boldsymbol{E}\left(e^{\alpha_{i} f-f_{t} \mid} / / \mathscr{F}_{t}\right)\right\|_{\infty}<+\infty \text {. }
$$

The above theorem, in fact, holds for all martingales, i.e., without the restrictive hypothesis $(\mathrm{H})$ (with an appropriately modified definition of BMO). It is only for simplicity that I introduced the hypothesis $(\mathrm{H})$ and also because this is the only case that we shall need for the applications. 
The above theorem in its "diadic martingale" (Paley-Walsh) version was known to J. Garnett even before they (together with Peter Jones) proved the general real variable theorem (G.J.).

My proof of Theorem G.J. Prob. is directly inspired from Garnett's original proof which is unpublished and which he gratiously put at my disposal.

The plan now is to prove Theorem G.J. Prob.; this is done in $\S 1$ and then to deduce from that Theorem G.J. This is done in $\S 2$. Before that however in the remainder of this paragraph I shall recall some standard facts and some notation of probability theory and on $\operatorname{BMO}\left(\boldsymbol{R}^{n}\right)$.

0.2. Notations and facts of probability theory. Let $\left(\Omega ; \mathscr{F} ; \mathscr{F}_{t}\right.$, $t \geqq 0, P$ ) be as above. We then say that $T$, a nonnegative (possibly $+\infty)$, r.v. is a stopping time if for all $t \geqq 0$ we have:

$$
[T \leqq t] \in \mathscr{F}_{t} ; \quad t \geqq 0
$$

With a stopping time $T$ we can associate then $\mathscr{F}_{T}$ a field of events (the events prior to $T$ ) defined by

$$
A \in \mathscr{F}_{T} \Longleftrightarrow A \cap[T \leqq t] \in \mathscr{F}_{t} ; \quad t \geqq 0 \text { ! }
$$

Observe that for two stopping times $T_{1} \leqq T_{2}$ we have $\mathscr{F}_{T_{1}} \subseteq \mathscr{F}_{T_{2}}$.

Let now $f \in L^{1}(\Omega)$ and let $f_{t}(t \geqq 0)$ be as in (0.1.3) and let us suppose, by hypothesis $(\mathrm{H})$, that we have already taken representatives of $f_{t}$ such that the trajectories $f_{t}(\omega)$ are continuous in $t$ (a.s. $\omega \in \Omega)$. Let us finally denote by $f_{\infty}=f$. For any stopping time $T$ we then have

$$
f_{T}=E\left(f / / \mathscr{F}_{T}\right) .
$$

$\left(f_{T}\right)(\omega)$ is of course defined to be $f_{T(\omega)}(\omega)$. The above is a basic fact of Martingale theory (cf. [10], IV. 5.5).

We shall finally find it convenient to adopt also the following abusive notation

$$
\boldsymbol{E}(f / / T)=\boldsymbol{E}\left(f / / \mathscr{F}_{T}\right)
$$

for any $f \in L^{1}(\Omega)$ and any stopping time $T$ and also

$$
E(A / \mathscr{G})=E[I(A) / / \mathscr{G}]
$$

for any event $A \subset \Omega$ and any $\mathscr{G} \subset \mathscr{F}$ subfield of $\mathscr{F}$, where $I(A)$ denotes the indicator function of $A$.

Let now $f \in \operatorname{BMO}(\Omega)$; there exist then positive constants such that 


$$
\begin{gathered}
\boldsymbol{E}\left[\left|f-f_{t}\right| / / \mathscr{F}_{t}\right] \leqq K_{1} \\
\boldsymbol{E}\left[e^{\alpha\left|f-f_{t}\right|} / / \mathscr{F}_{t}\right] \leqq K_{2}
\end{gathered}
$$

(0.2.2) is the definition of $\operatorname{BMO}(\Omega)$ and (0.2.3) is the John-Nirenberg theorem which also holds for continuous time martingales (cf. [9], p. 348. The proof is in fact even easier for continuous time!).

Let then $T$ be a stopping time and $f \in \operatorname{BMO}(\Omega)$. It is easy to verify that if $f$ satisfies $(0.2 .2)$ then

$$
E\left[\left|f-f_{T}\right| / / T\right] \leqq K_{1}
$$

and if $f$ satisfies $(0.2 .3)$ then

$$
\boldsymbol{E}\left[e^{\alpha\left|f-f_{T}\right|} / / T\right] \leqq K_{2} .
$$

Let us also denote by

$$
\varphi=f^{T}=f-f_{T}
$$

we then have:

$$
[\varphi \neq 0] \subset[T<+\infty]
$$

and it is easy to verify that if $f$ satisfies (0.2.2) for some $K_{1}$ then $\varphi=f^{T}$ satisfies (0.2.2) also with the same $K_{1}$. Similarly if $f$ satisfies (0.2.3) for some $K_{2}$ then $\varphi=f^{T}$ also satisfies (0.2.3) with the same $K_{2}$.

The standard way to prove all the above assertions is to verify them first under the additional assumption that $T$ takes only finitely many values, and then to write a general stopping time $T$ as the limit of a nonincreasing sequence of such special stopping times.

One final fact will be needed:

Lemma 0.1. Let $f \in L^{1}(\Omega)$ and let us suppose that for all $t \geqq 0$ there exists $a_{t} \in L^{1}(\Omega)$ which is measurable with respect to the subfield $\mathscr{F}_{t}$ and which satisfies

$$
\boldsymbol{E}\left[\left|f-a_{t}\right| / / \mathscr{F}_{t}\right] \leqq K
$$

for some constant $K$. It then follows that $f \in \mathrm{BMO}(\Omega)$ and that $\|f\|_{\text {вмо }} \leqq 2 K$.

The proof is obvious.

0.3. Notations and facts from real variable theory. Let $z=$ $(x, y) \in \boldsymbol{R}_{+}^{n+1}, x \in \boldsymbol{R}^{n}, y>0$ and let us denote by

$$
P_{z} f=c_{n} \int_{t \in \boldsymbol{R}^{n}} \frac{y f(t) d t}{\left(y^{2}+\|x-t\|^{2}\right)^{(n+1) / 2}}
$$


the Poisson integral of $f \in L_{10 c}^{1}\left(\boldsymbol{R}^{n}\right)$ evaluated at $z$, i.e., the value at $z$ of the harmonic extension of $f$ on the upper half space (this under the assumption that the integral in (0.3.1) converges absolutely (cf. [11]). Let then $f \in \mathrm{BMO}\left(\boldsymbol{R}^{n}\right)$; it is an easy matter to verify that:

$$
\sup _{z \in \boldsymbol{R}_{+}^{n+1}} P_{z}\left[\left|f-P_{z} f\right|\right]<+\infty \text {. }
$$

This fact is well known.

What is perhaps a little less evident is that if $f \in \operatorname{BMO}\left(\boldsymbol{R}^{n}\right)$ and $\alpha>0$ are such that (0.1.2) is verified then:

$$
\sup _{z \in \boldsymbol{R}_{+}^{n+1}} P_{z}\left[e^{\beta\left|f-P_{z} f\right|}\right]<+\infty, \quad \forall \beta<\frac{\alpha}{n} .
$$

Observe that the situation both in (0.3.2) and (0.3.3) is dilation and translation invariant and this means that we can suppose that $z=$ $i=(0,1)$. For $z=i$ then the proof of both (0.3.2) and (0.3.3) is based on the following elementary inequalities that will be stated without proof.

$$
P_{i} F \leqq C \sum_{j \geqq 1} 2^{-(n+1) j} \int_{\left|x_{k}\right| \leqq 2^{j}} F d x_{1} \cdots d x_{n}
$$

for all $F \geqq 0$ where $C$ is a constant that only depends on $n$.

$$
\left\|P_{i} f-f_{I_{1}}\right\| \leqq C\|f\|_{\text {вмо }}
$$

for all $f \in \operatorname{BMO}\left(\boldsymbol{R}^{n}\right)$ where $I_{1}$ is the unit cube of $\boldsymbol{R}^{n}$ centered at the origin and where $C$ is as above.

Finally if $f \in$ BMO satisfies (0.1.2) for some $\alpha>0$ then

$$
e^{\alpha\left|f_{I}-f_{J}\right|} \leqq K \frac{|J|}{|I|}
$$

for any two cubes $I$ and $J$ with sides parallel to the axes such that $I \subset J$ where now $K$ depends both on $n$ and on $\alpha$.

The proof of both (0.3.2) and (0.3.3) can now be supplied by the reader (I hope).

1. Proof of Theorem G.J. Prob. In this paragraph we shall fix once and for all a probability space $(\Omega, \mathscr{F}, P)$ and $\left(\mathscr{F}_{t}, t \geqq 0\right)$ a family of subfields that satisfy the conditions introduced in $\S 0.1$, in particular hypothesis $(\mathrm{H})$.

1.1. Graded sequences of stopping times. Let $0=T_{0} \leqq T_{1} \leqq \cdots \leqq$ $T_{n} \leqq \cdots$ be a nondecreasing sequence of stopping times. We shall say that the above sequence is $\gamma$-Graded for some $0<\gamma<1$ if 


$$
\boldsymbol{E}\left[\left(T_{i+1}<+\infty\right) / / T_{i}\right] \leqq \gamma ; \quad i=0,1, \cdots .
$$

We have then:

Proposition 1.1. Let $\mathscr{T}=\left(T_{i} ; i \geqq 0\right)$ be a $\gamma^{m}$-Graded sequence of stopping times for some $0<\gamma<1$ and some positive integer $m$. There exists then $\mathscr{S}=\left(S_{i} ; i \geqq 0\right)$ a $\gamma$-Graded sequence of stopping times s.t. $T_{j}=S_{m j}, j=0,1, \cdots$.

The above proposition follows immediately from the following.

Lemma 1.1. Let $\gamma$ and $m$ be as in Proposition 1.1 and let $R \leqq S$ be two stopping times on $\Omega$ s.t.

$$
\boldsymbol{E}[(\boldsymbol{S}<+\infty) / / R] \leqq \gamma^{m} .
$$

There exists then a sequence of stopping times $\left(T_{i}, i=0,1, \cdots, m\right)$ s.t.

$$
R=T_{0} \leqq T_{1} \leqq \cdots \leqq T_{m}=S
$$

and

$$
E\left[\left(T_{i+1}<+\infty\right) / / T_{i}\right] \leqq \gamma ; \quad i=0,1, \cdots, m-1 .
$$

Proof. Let us denote:

$f_{t}=\boldsymbol{E}\left[(S<+\infty) / / \mathscr{F}_{t}\right], \quad t \geqq 0 ; \quad f_{\infty}=f=I[S<+\infty] ;$

and let us define stopping times:

$$
T_{i}=\inf \left\{t ; f_{t}=\gamma^{m-i}\right\} ; \quad i=1,2, \cdots, m-1 .
$$

The claim is that $R=T_{0}, T_{1}, \cdots, T_{m-1}, T_{m}=S$ satisfy the conditions of the lemma. Indeed (1.1.2) and (0.2.1) imply that

$$
f_{R} \leqq \gamma^{m} ; \quad f_{S}=I(S<+\infty)
$$

and this implies (1.1.3) at once. On the other hand the definition of the $T_{i}$ 's and (0.2.1) imply that:

$$
\begin{aligned}
& \boldsymbol{E}\left(f / / T_{i}\right)=\gamma^{m-i} \boldsymbol{I}\left[T_{i}<+\infty\right] ; \quad i=1,2, \cdots, m-1 \\
& \boldsymbol{E}\left(f / / T_{0}\right) \leqq \gamma^{m} \boldsymbol{I}\left[T_{0}<+\infty\right] .
\end{aligned}
$$

If we substitute the above in the relation

$$
\boldsymbol{E}\left(f / / T_{i}\right)=\boldsymbol{E}\left[\boldsymbol{E}\left(f / / T_{i+1}\right) / / T_{i}\right] ; \quad i=0,1, \cdots, m-1
$$

we obtain:

$$
\gamma^{m-i-1} \boldsymbol{E}\left[\left(T_{i+1}<+\infty\right) / / T_{i}\right] \leqq \gamma^{m-i} I\left[T_{i}<+\infty\right]
$$


A PROBABILISTIC PROOF OF THE GARNETT-JONES THEOREM ON BMO 207 $i=0,1, \cdots, m-1$; this proves (1.1.4) and completes the proof of the lemma.

1.2. Graded Functions. Let $f \in L^{1}(\Omega)$ and $0<\gamma<1$. We shall then say that $f$ is a $\gamma$-Graded function if there exists a $\gamma$-Graded sequence of stopping times $\left(T_{i} ; i \geqq 0\right)$ s.t.

$$
f=\sum_{i=1}^{\infty} I\left[T_{i}<+\infty\right] \text {. }
$$

We have then:

Proposition 1.2. If $f \in L^{1}(\Omega)$ is a $\gamma$-Graded function then $f \in \mathrm{BMO}$ and $\|f\|_{\text {вмо }} \leqq 2 /(1-\gamma)$.

Proof. Let $\left(T_{i} ; i \geqq 0\right)$ be a $\gamma$-Graded sequence of stopping times s.t.

$$
f=\sum_{i=1}^{\infty} I\left[T_{i}<+\infty\right] \text {. }
$$

For all $t \geqq 0$ and $n=0,1, \cdots$ let us then define

$$
\begin{gathered}
a_{t}=\sum_{i \geqq 1 ; T_{i} \leqq t} I\left(T_{i}<+\infty\right) \\
X_{t}^{n}=\left\{\omega \in \Omega \mid T_{0} \leqq t, \cdots, T_{n} \leqq t, T_{n+1}>t\right\} .
\end{gathered}
$$

The following facts are then clear (cf. (1.1.1)):

$$
\begin{gathered}
{\left[a_{t}=n\right]=X_{t}^{n} \in \mathscr{F}_{t} ; \quad t \geqq 0, \quad n=0,1,2, \cdots} \\
P\left(\bigcup_{n} X_{t}^{n}\right)=1 ; \quad t \geqq 0 .
\end{gathered}
$$

For all $n \geqq 1$ and $t \geqq 0$ we also have

$$
\begin{aligned}
\boldsymbol{E}\left[\left|f-a_{t}\right| / / \mathscr{F}_{t}\right] \boldsymbol{I}\left(X_{t}^{n-1}\right) & =\boldsymbol{E}\left[\left|f-a_{t}\right| \boldsymbol{I}\left(X_{t}^{n-1}\right) / / \mathscr{F}_{t}\right] \boldsymbol{I}\left(X_{t}^{n-1}\right) \\
& =\boldsymbol{E}\left[\left(\sum_{j=n}^{\infty} \boldsymbol{I}\left(T_{j}<+\infty\right)\right) \boldsymbol{I}\left(X_{t}^{n-1}\right) / / \mathscr{F}_{t}\right] \boldsymbol{I}\left(X_{t}^{n-1}\right) \\
& =\boldsymbol{E}\left\{\boldsymbol{E}\left[\left(\sum_{j=n}^{\infty} \boldsymbol{I}\left(T_{j}<+\infty\right)\right) / / T_{n}\right] / / \mathscr{F}_{t}\right\} \boldsymbol{I}\left(X_{t}^{n-1}\right)
\end{aligned}
$$

because $T_{n}>t$ on $X_{t}^{n-1}$, and because $X_{t}^{n-1}=\left[T_{n-1} \leqq t\right] \cap\left[T_{n}>t\right] \in \mathscr{F}_{T_{n}}$. But:

$$
\begin{aligned}
\boldsymbol{E}\left[\left(\sum_{j=n}^{\infty} \boldsymbol{I}\left(T_{j}<+\infty\right)\right) / / T_{n}\right] & =\sum_{j=n}^{\infty} \boldsymbol{E}\left[\left(T_{j}<+\infty\right) / / T_{n}\right] \\
& \leqq 1+\gamma+\gamma^{2}+\cdots=\frac{1}{1-\gamma}
\end{aligned}
$$


a direct substitution of (1.2.3) into the previous relation, together with (1.2.2) implies therefore that:

$$
E\left[\left|f-a_{t}\right| / / \mathscr{F}_{t}\right] \leqq \frac{1}{1-\gamma}
$$

and this together with Lemma 0.1 (which can be applied here because of (1.2.1), completes the proof of Proposition 1.2.

The above proposition admits a converse which presents some independent interest.

THEOREM 1.2. Let $F$ be a real valued function in $\mathrm{BMO}(\Omega)$ and let $\alpha>0$ and $K$ be such that:

$$
E\left[\exp \left(\alpha\left|F-F_{t}\right|\right) / / \mathscr{F}_{t}\right] \leqq K, \quad t \geqq 0 .
$$

Then for all $\lambda \geqq \lambda_{0}(\alpha, K)$ [some explicit positive function of $\alpha$ and $K$ that will be determined in the proof] there exists a decomposition of $F$ :

$$
F=\lambda \Phi^{+}-\lambda \Phi^{-}+\Psi+\boldsymbol{E}(\boldsymbol{F})
$$

where $\Psi \in L^{\infty}(\Omega)$ and $\|\Psi\|_{\infty} \leqq \lambda$ and where the two functions $\Phi^{+}$and $\Phi^{-}$are $e^{-\alpha \lambda / 2}$ Graded on $\Omega$.

The proof of the theorem depends on the following elementary but fairly lengthy combinatorial:

LemmA 1.2. Let $0<\gamma<1 / 2$ and let

$$
0=T_{0} \leqq T_{1} \leqq \cdots \leqq T_{n} \leqq \cdots
$$

be a nondecreasing sequence of stopping times; let further

$$
A_{i} \subseteq\left[T_{i}<+\infty\right] ; \quad A_{i} \in \mathscr{F}_{T_{i}}, \quad i=1,2, \cdots
$$

be a sequence of events s.t.

$$
\boldsymbol{E}\left(A_{i+1} / / T_{i}\right) \leqq \gamma ; \quad i=0,1, \cdots .
$$

Then the function

$$
F=\sum_{i=1}^{\infty} \boldsymbol{I}\left(A_{i}\right)
$$

ia a $\gamma /(1-\gamma)$-Graded function.

Proof. (cf. Addendum for a simpler proof). The proof will require a number of notations.

Let us denote by $\mathscr{I}_{n}(n \geqq 1)$ the set of increasing multi-indices 
i.e., $I \in \mathscr{F}_{n}$ if

$$
I=\left(i_{1}, i_{2}, \cdots, i_{n}\right) ; \quad 1 \leqq i_{1}<i_{2}<\cdots<i_{n} .
$$

For $I \in \mathscr{F}_{n}$ as above we shall denote by $m(I)=i_{n}$ its largest element and for any $r, 1 \leqq r \leqq n$ we shall denote by

$$
I_{r}=\left(i_{1}, \cdots, i_{r}\right) \in \mathscr{F}_{r}
$$

the multi-index obtained by truncating $I$ at $r$.

For every fixed $n$ we shall give to $\mathscr{F}_{n}$ its lexicographical total order i.e., for $I, J \in \mathscr{F}_{n}$ we shall say

$$
I=\left(i_{1}, \cdots, i_{n}\right)<J=\left(j_{1}, \cdots, j_{n}\right)
$$

if for some $r, 1 \leqq r \leqq n$ we have $i_{1}=j_{1}, \cdots, i_{r-1}=j_{r-1}, i_{r}<j_{r}$. For every fixed $I \in \mathscr{F}_{n}$ let us then denote by:

$$
A_{I}=\bigcap_{i \in I} A_{i} ; \quad X_{I}=A_{I} \backslash \bigcup_{\substack{j \in I \\ j \leqq m(I)}} A_{j}
$$

and for all $m \geqq 1$ let us denote by

$$
B_{n}=\bigcup_{I \in \cup n} A_{I}
$$

It is evident that for all $J, J<I$ we have

$$
A_{J} \subset \bigcup_{\substack{j \notin I \\ j \leqq m(I)}} A_{j}
$$

This implies that the sets $\left\{X_{I} ; I \in \mathscr{F}_{n}\right\}$ are disjoint. On the other hand:

$$
B_{n}=\bigcup_{I \in Y_{n}} X_{I} \quad \text { (disjoint union). }
$$

Indeed let $\omega \in B_{n}$ and let $I$ be the first multi-index in $\mathscr{J}_{n}$ (first in the order of $\mathscr{F}_{n}$ ) such that

$$
\omega \in A_{I}
$$

It is then clear that for all $j \notin I, j \leqq m(I)$ we have:

$$
\omega \notin A_{j} \text {. }
$$

For if $\omega \in A_{j}$ for some such $j$ we could then construct a multi-index $I^{\prime} \in \mathscr{F}_{n}$ that is strictly smaller than $I$ and s.t. $\omega \in A_{I^{\prime}}$. The above sets satisfy:

$$
\begin{gathered}
X_{I} \in \mathscr{F}_{T_{m(I)}} \\
X_{I} \subset X_{I_{r}} ; \quad \forall n, r, \quad 1 \leqq r \leqq n ; \quad \forall I \in \mathscr{F}_{n} .
\end{gathered}
$$


(1.2.5) follows from the hypothesis and the definition of the $X_{I}$ 's. To prove (1.2.6) observe that we always have:

$$
X_{I} \subset A_{I_{r}}, \quad \forall n, r, \quad 1 \leqq r \leqq n ; \quad \forall I \in \mathscr{I}_{n} .
$$

But also if we fix $I=\left(i_{1}, \cdots, i_{n}\right) \in \mathscr{J}_{n}$ and some $r, 1 \leqq r \leqq n$ then

$$
X_{I} \cap A_{j}=\varnothing, \quad \forall j \notin I_{r}, \quad j \leqq i_{r}
$$

by the definition of $X_{I}$; this together with (1.2.7) implies (1.2.6). (1.2.6) and the fact that the $X_{I}$ 's, $I \in \mathscr{J}_{n}$ are disjoint implies that

$$
B_{n+1} \cap X_{I}=\bigcup_{J \in \mathcal{S}_{n+1} ; J_{n}=I} X_{J} ; \quad n \geqq 1, \quad I \in \mathscr{I}_{n} .
$$

For every fixed $n \geqq 1$ let us now define a r.v.

$$
S_{n}(\omega)=\left\{\begin{array}{lll}
T_{m(I)}(\omega) & \text { if } \omega \in X_{I}, \quad I \in \mathscr{I}_{n} \\
+\infty & \text { if } \omega \notin B_{n} .
\end{array}\right.
$$

It is clear then that:

$$
\left[S_{n}<+\infty\right]=B_{n} ; \quad\left[S_{n} \leqq t\right]=\bigcup_{I \in \cup_{n}}\left[X_{I} \cap\left(T_{m(I)} \leqq t\right)\right] .
$$

From this and (1.2.5) it follows that $S_{n}(n \geqq 1)$ is a stopping time. On the other hand if $1 \leqq p<q$ and if $\omega \in B_{q}$ there exists a unique $I \in \mathscr{J}_{q}$ s.t. $\omega \in X_{I}$ but this implies that $\omega \in X_{I_{p}}$ by (1.2.6) and therefore that:

$$
S_{q}(\omega)=T_{m(I)}(\omega) \geqq T_{m\left(I_{p}\right)}(\omega)=S_{p}(\omega)
$$

so that we have:

$$
0=S_{0} \leqq S_{1} \leqq \cdots \leqq S_{n} \leqq \cdots
$$

It is finally clear that

$$
F=\sum_{j=1}^{\infty} I\left(A_{j}\right)=\sum_{j=1}^{\infty} I\left(B_{j}\right)=\sum_{j=1}^{\infty} I\left(S_{j}<+\infty\right)
$$

by (1.2.9); therefore to prove our lemma it suffices to show that:

$$
\xi_{n}=E\left[\left(S_{n+1}<+\infty\right) / / S_{n}\right] \leqq \frac{\gamma}{1-\gamma} ; \quad n=0,1, \cdots .
$$

Assume first that $n \geqq 1$. We have then

$$
\xi_{n} I\left(\complement B_{n}\right)=E\left[\left(S_{n+1}<+\infty\right) \cap \complement B_{n} / / S_{n}\right]=0, \quad\left(\mathcal{G} B_{n}=\Omega \backslash B_{n}\right) .
$$

On the other hand for every fixed $I \in \mathscr{F}_{n}$ we have $X_{I} \in \mathscr{F}_{s_{n}}$ by (1.2.9) and this implies that (cf. (1.2.8)): 
A PROBABILISTIC PROOF OF THE GARNETT-JONES THEOREM ON BMO 211

$$
\begin{aligned}
& \xi_{n} I\left(X_{I}\right)=E\left(B_{n+1} \cap X_{I} / / T_{m(I)}\right) \leqq \sum_{\substack{J \in J_{n+1} \\
J_{n}=I}} E\left(A_{J} / / T_{m(I)}\right) \\
& \leqq \sum_{j=m(L)+1}^{\infty} \boldsymbol{E}\left(A_{j} / / T_{m(I)}\right) \leqq \gamma+\gamma^{2}+\cdots=\frac{\gamma}{1-\gamma} .
\end{aligned}
$$

This together with (1.2.5) and (1.2.11) proves (1.2.10) for $n \geqq 1$. For $n=0$ we have

$$
\xi_{0}=E\left(B_{1}\right)=\sum_{j=1}^{\infty} E\left(X_{j}\right) \leqq \sum_{j=1}^{\infty} E\left(A_{j}\right) \leqq \gamma+\gamma^{2}+\cdots=\frac{\gamma}{1-\gamma}
$$

(1.2.6) therefore always holds. This completes the proof of Lemma 1.2 .

We can now give the:

Proof of Theorem 1.2. Let $\alpha, K, F$ be as in the theorem and let us choose $\lambda_{0}$ s.t.

$$
K e^{-\alpha \lambda}\left(1-K e^{-\alpha \lambda}\right)^{-1} \leqq e^{-\alpha \lambda / 2} ; \quad \forall \lambda \geqq \lambda_{0} .
$$

Let also $\lambda>\lambda_{0}$ be fixed. We shall determine then a sequence of stopping times

$$
0=T_{0} \leqq T_{1} \leqq \cdots \leqq T_{n} \leqq \cdots
$$

and a sequence of functions

$$
F^{(i)} \in L^{1}(\Omega) ; \quad i=1,2, \cdots
$$

by the following relations:

$$
\begin{gathered}
F^{(1)}=F-E(F) \\
T_{i}=\inf \left\{t ;\left|F_{t}^{(i)}\right| \geqq \lambda\right\} \\
F^{(i+1)}=F^{(i)}-E\left(F^{(i)} / \mid T_{i}\right)
\end{gathered}
$$

for $i=1,2, \cdots$.

It is clear that:

$$
F_{t}^{(i+1)}=F_{t}^{(i)}-F_{T_{i} \wedge t}^{(i)}
$$

and this implies that $F_{t}^{(i+1)}=0$ if $t \leqq T_{i}$ so that the sequence of stopping times $\left(T_{i} ; i \geqq 0\right)$ so constructed is indeed increasing. By the remarks made in $\S 0.1$ we then have:

$$
\begin{aligned}
& \boldsymbol{E}\left[\exp \left(\alpha\left|F^{(i)}-F_{t}^{(i)}\right|\right) / / \mathscr{F}_{t}\right] \leqq K \\
& \boldsymbol{E}\left[\exp \left(\alpha\left|F^{(i)}\right|\right) / / T_{i-1}\right] \leqq K
\end{aligned}
$$

for all $i=1,2, \cdots$.

By Jensen's convexity inequality we also have: 


$$
\begin{aligned}
\boldsymbol{E}\left[\exp \alpha\left|F^{(i)}\right| / / T_{i}\right] & \geqq \exp \left[\alpha \boldsymbol{E}\left(\left|F^{(i)}\right| / / T_{i}\right)\right] \\
& \geqq \exp \alpha\left|\boldsymbol{E}\left(F^{(i)} / / T_{i}\right)\right|=\exp \alpha\left|F_{T_{i}}^{(i)}\right| \\
& \geqq e^{\alpha \lambda} \boldsymbol{I}\left[T_{i}<+\infty\right] .
\end{aligned}
$$

This implies that:

$$
\begin{aligned}
\boldsymbol{E}\left[\exp \left(\alpha\left|F^{(i)}\right|\right) / / T_{i-1}\right] & =\boldsymbol{E}\left\{\boldsymbol{E}\left[\exp \left(\alpha\left|F^{i}\right|\right) / / T_{i}\right] / / T_{i-1}\right\} \\
& =e^{\alpha \lambda} \boldsymbol{E}\left[\left(T_{i}<+\infty\right) / / T_{i-1}\right]
\end{aligned}
$$

and this together with (1.2.12) implies that

$$
E\left[\left(T_{i}<+\infty\right) / / T_{i-1}\right] \leqq K e^{-\alpha \lambda}=\gamma ; \quad i=1,2, \cdots
$$

i.e., that the sequence $\left(T_{i} ; i \geqq 0\right)$ is $\gamma$-Graded, $(\gamma<1$ by our hypothesis on $\lambda$ ).

We shall need the following further facts about the above functions

$$
\begin{gathered}
F^{(n)} \underset{n \rightarrow \infty}{\longrightarrow} 0 \\
\left|\sum_{i=1}^{n} F_{T_{i}}^{(i)} I\left[T_{i}=+\infty\right]\right| \leqq \lambda ; \quad n=1,2, \cdots .
\end{gathered}
$$

(1.2.13) follows from the fact

$$
\left[F^{(i)} \neq 0\right] \subset\left[T_{i-1}<+\infty\right] ; \quad i=1,2, \cdots
$$

[cf. (0.2.4)] and the fact that the $T_{i}$ 's are $\gamma$-Graded. To prove (1.2.14) observe that the general term of the summation is

$$
\alpha_{i}=F_{T_{i}}^{(i)} I\left[T_{i}=+\infty\right]
$$

and satisfies

$$
\left[\alpha_{i} \neq 0\right] \subset\left[T_{i}=+\infty\right] \cap\left[T_{i-1}<+\infty\right]
$$

so that $\alpha_{i} \alpha_{j}=0$ for all $i \neq j$. (1.2.14) then follows from the fact

$$
\left|F_{T_{i}}^{(i)}\right| \leqq \lambda
$$

(by the definition of $T_{i}$ ).

We can now complete the proof of our theorem. Indeed we have

$$
F=\sum_{i=1}^{n} \boldsymbol{E}\left(F^{(i)} / / T_{i}\right)+F^{(n+1)}+\boldsymbol{E}(F)
$$

for all $n \geqq 1$. Letting $n \rightarrow \infty$ and taking into account (1.2.13) we obtain therefore that:

$$
F=\sum_{i=1}^{\infty} F_{T_{i}}^{(i)} I\left[T_{i}<+\infty\right]+\Psi+E(F)
$$


A PROBABILISTIC PROOF OF THE GARNETT-JONES THEOREM ON BMO 213

where:

$$
\Psi=\sum_{i=1}^{\infty} F_{T_{i}}^{(i)} I\left[T_{i}=+\infty\right]
$$

and where $\|\Psi\|_{\infty} \leqq \lambda$ by (1.2.14)

Let us then set

$$
\begin{aligned}
& A_{i}^{+}=\left\{\omega ; T_{i}(\omega)<+\infty F_{T_{i}}^{(i)}=\lambda\right\} \\
& A_{i}^{-}=\left\{\omega ; T_{i}(\omega)<+\infty F_{T_{i}}^{(i)}=-\lambda\right\} \\
& \Phi^{+}=\sum_{i=1}^{\infty} I\left(A_{i}^{+}\right) ; \quad \Phi^{-}=\sum_{i=1}^{\infty} I\left(A_{i}^{-}\right) .
\end{aligned}
$$

It then follows that:

$$
F=\lambda \Phi^{+}-\lambda \Phi^{-}+\Psi+E(F) .
$$

The sets $\left(A_{i}^{+} ; i \geqq 1\right)$ and $\left(A_{i}^{-} ; i \geqq 1\right)$ on the other hand satisfy the conditions of Lemma 1.2 for the stopping times $\left(T_{i} ; i \geqq 0\right)$. The Lemma 1.2 and the choice of $\lambda_{0}$ therefore implies that the two functions $\Phi^{+}$and $\Phi^{-}$are $e^{-\alpha \lambda / 2}$-Graded. This completes the proof of the theorem.

1.3. Proof of Theorem G.J. Prob. We shall need the following.

LEMMA 1.3. Let $0<\gamma<1$ and $m$ a positive integer. Let also $\Phi$ be a $\gamma^{m}$-Graded function. Then there exists $\Theta$ a $\gamma$-Graded function s.t. $\Phi-(1 / m) \Theta \in L^{\infty}$.

Proof. Indeed if $\mathscr{T}=\left(T_{i} ; i \geqq 0\right)$ is a $\gamma^{m}$-Graded sequence of stopping times such that

$$
\Phi=\sum_{i=1}^{\infty} I\left[T_{i}<+\infty\right]
$$

and if by Proposition $1.1, \mathscr{S}=\left(S_{i} ; i \geqq 0\right)$ is a $\gamma$-Graded sequence of stopping times such that $S_{m j}=T_{j}(j=0,1, \cdots)$ then clearly

$$
\left|\Phi-\frac{1}{m} \sum_{i=1}^{\infty} I\left[S_{i}<+\infty\right]\right| \leqq 1 \text {. }
$$

This proves the lemma. We shall now give the:

Proof of Theorem G.J. Prob. Let $F \in \mathrm{BMO}(\Omega)$ and let us suppose, as we may, that $F$ is real, that $\boldsymbol{E}(F)=0$ and that $\alpha>0$ an $K$ are such that

$$
\boldsymbol{E}\left(\exp \left(\alpha\left|F-F_{t}\right|\right) / / \mathscr{F}_{t}\right) \leqq K, \quad t \geqq 0
$$


Let us fix some $\lambda \geqq \lambda_{0}(\alpha, K) \quad\left(\lambda_{0}(\alpha, K)\right.$ is the function defined in Theorem 1.2) such that $\lambda \alpha=m$ is a positive integer and let us decompose

$$
F=\lambda \Phi^{+}-\lambda \Phi^{-}+\Psi
$$

as in Theorem 1.2. If we set then $\gamma=e^{-1 / 2}$ we see that both the functions $\Phi^{+}$and $\Phi^{-}$are $\gamma^{m}$-Graded; it follows therefore by Lemma 1.3 that there exist two $\gamma$-Graded functions $\Theta^{+}$and $\Theta^{-}$such that:

$$
\left|\Phi^{+}-\frac{1}{m} \Theta^{+}\right| \leqq 1 ; \quad\left|\Phi^{+}-\frac{1}{m} \Theta^{-}\right| \leqq 1 .
$$

This implies that:

$$
F-\frac{\lambda}{m} \Theta^{+}+\frac{\lambda}{m} \Theta^{-}=F-\frac{1}{\alpha} \Theta^{-}+\frac{1}{\alpha} \Theta^{-} \in L^{\infty}
$$

and since by Proposition 1.2

$$
\left\|\Theta^{+}\right\|_{\text {вмо }},\left\|\Theta^{-}\right\|_{\text {вмо }} \leqq \frac{2}{1-\gamma}=\frac{2 \sqrt{e}}{\sqrt{e}-1}
$$

we conclude that $d\left(F, L^{\infty}\right)$ the distance of $F$ from $L^{\infty}$ in BMO satisfies

$$
d\left(F, L^{\infty}\right) \leqq \frac{10}{\alpha}
$$

which is the nontrivial inequality in Theorem G.J. Prob. This completes the proof.

2. Proof of the Garnett-Jones Theorem. I shall prove Theorem G.J. in the setting of the unit circle (i.e., the periodic case $\boldsymbol{R} \bmod (2 \pi))$. The reason is that Brownian motion in the unit disc has a natural starting point, namely the origin. The proof, of course, readily generalizes to $\boldsymbol{R}^{n}$ by considering Brownian motion starting from some appropriately high level (cf. [2]; [9], p. 129 of $X)$ in $\boldsymbol{R}_{+}^{n+1}$. The reader who possesses some technique can do that for himself without any difficulty, I am sure. (cf. Addendum for an alternative approach).

2.1. Brownian motion in the unit disc. Let $b_{1}(t), b_{2}(t)(t \geqq 0$; $\left.b_{i}(0)=0, i=1,2\right)$ be two independent copies of standard Brownian motion starting at the origin and let us denote by

$$
z(t)=z_{t}(\omega)=b_{1}(t)+i b_{2}(t) \in C
$$

which we shall call Brownian motion in the plane starting at the origin, or simply complex Brownian motion. Let us also define 


$$
\mathscr{F}_{t}=\{\sigma \text {-field generated by } z(s), s \leqq t\} \text {. }
$$

It is then well known (cf. [5], [9], p. 286 of $X$ ) that the probability space and the $\sigma$-algebras that we obtain $\left(\Omega ; \mathscr{F} ; \mathscr{F}_{t}(t \geqq 0) ; P\right)$ satisfy the hypothesis $(\mathrm{H})$.

We shall need to apply the Markov property so we recall here some of the standard notations and facts of Markov processes (cf. [6], especially 2.5). The way to define the Markov process underlying complex Brownian motion is to set for $\Omega$ the space of all paths (and not only the ones that start at the origin). In that space $\Omega$ we have then

$$
\theta_{t}: \Omega \longrightarrow \Omega
$$

the shift operator that is defined by $\left[\theta_{t}(\omega)\right]_{s}=\omega_{t+s},\left[\omega_{\alpha} \in C\right.$ is the position of the path $\omega$ at time $\alpha \geqq 0]$. A family of probabilities is also defined $\left(\boldsymbol{P}_{a} ; a \in \boldsymbol{C}\right)$ on $\Omega$ that is connected by the ChapmanKolmogorov Equations. $\quad\left(\boldsymbol{P}_{a}\right.$ is the probability that controls Brownian motion starting from $a \in \boldsymbol{C}$.) With our previous notations we have of course $\boldsymbol{P}_{0}=\boldsymbol{P}$. The Markov property of Brownian motion can then be expressed by

$$
\boldsymbol{E}_{0}\left[G\left[\theta_{t}(\omega)\right] / / \mathscr{F}_{t}\right]=\boldsymbol{E}_{a}[G(\omega)], \quad a=z_{t} ; \quad \boldsymbol{P}_{0} \quad \text { a.s. }
$$

for any bounded Borel function $G$ on $\Omega$. $\boldsymbol{E}_{a}$ denotes, of course, the expectation associated with $\boldsymbol{P}_{a}$ (cf. [6], § 2.5).

We shall now define $T$ a nonnegative function on $\Omega$ by

$$
T=\inf \left\{t ;\left|\omega_{t}\right| \geqq 1\right\}
$$

which is the hitting time of the path $\omega$ on the complement of the unit disc $D=\{|z| \leqq 1\}$ and it satisfies:

$$
t+T\left[\theta_{t}(\omega)\right]=T(\omega) \text { on the set }[T \geqq t]
$$

(cf. [1], Chapter I, $\S 10)$. We finally have $z_{t}(\omega)=\omega_{t}\left(\boldsymbol{P}_{0}\right.$ a.e.).

2.2. The link between Brownian motion and analysis. We shall denote here by $\boldsymbol{T}=\boldsymbol{R}(\bmod 2 \pi)$ the unit circle. Let us first define

$$
M: C(T) \longrightarrow L^{\infty}(\Omega ; P)
$$

a linear operator by:

$$
M f=X \in L^{\infty}(\Omega), \quad X(\omega)=f\left(z_{T(\omega)}(\omega)\right) ; \quad \forall f \in C(T) .
$$

Using the fact that the hitting probability of Brownian motion on $\partial D=T$ is Lebesgue measure $\left(\boldsymbol{P}\left(z_{T} \in E\right)=1 / 2 \pi\right.$ Leb. measure of $E$ for all $E \subset \partial D$ ), it is easy to see that $M$ extends to a mapping 
$M: L^{p}(\boldsymbol{T}) \rightarrow L^{p}(\Omega)(1 \leqq p \leqq+\infty)$ and that $\|M f\|_{p} \leqq\|f\|_{p}\left(\forall f \in L^{p}(\boldsymbol{T})\right.$ $\forall p, 1 \leqq p \leqq+\infty)$. The main link between Brownian motion and probability is supplied by the following fact. Let $u \in C(D)$ be a function continuous on the closed disc and harmonic on $\dot{D}$, and let us denote by $X=M\left(\left.u\right|_{\partial D}\right)$. We have then:

$$
X_{t}=\boldsymbol{E}\left(X / / \mathscr{F}_{t}\right)=u(z(T \wedge t)) \text {. }
$$

This is but a variant of $\mathrm{S}$. Kakutani's celebrated theorem that says that if "you compose Brownian motion with a harmonic function you get a Brownian Martingale"; at any rate, for the above and other information on potential theory cf. [6], 2.19.

For $u$ and $X$ as in (2.1.3) we then have:

$$
\left\|\sup X_{t}\right\|_{L^{1}(\Omega)} \leqq C\left\|\sup _{0 \leqq r<1} u\left(r e^{i \theta}\right)\right\|_{L^{1}(T)} .
$$

This is a well known fact and is contained in [2] and [3]. With $u$ and $X$ again as in (2.1.3) we also have:

$$
\begin{gathered}
\|X\|_{\text {BMO }(\Omega)} \leqq \sup _{z \in D} P_{z}\{|u(\theta)-u(z)|\} \\
\sup _{t}\left\|E\left(e^{\alpha\left|X-X_{t}\right|} / \mid \mathscr{F}_{t}\right)\right\|_{\infty} \leqq \sup _{z \in \dot{D}} P_{z}\left[e^{\alpha|u(\theta)-u(z)|}\right]
\end{gathered}
$$

for all $\alpha>0, P_{z}[f(\theta)]$ denotes here the Poisson integral of the function $f=f(\theta)(\theta \in \boldsymbol{T})$ evaluated at the point $z \in \dot{D}$.

Both the above inequalities follow from the following more general inequality

$$
\begin{aligned}
\sup _{t} E\left\{\Phi[u(z(T))-u(z(T \wedge t))] / / \mathscr{F}_{t}\right\} \\
\leqq \max \left\{\Phi(0) ; \sup _{z \in \dot{D}} P_{z}(\Phi[u(\theta)-u(z)])\right\}
\end{aligned}
$$

where $\Phi(\xi)$ is a nonnegative continuous function of $\xi \in \boldsymbol{C}$. To prove the above inequality observe that

$$
\begin{aligned}
\boldsymbol{E}\left[\Phi / / \mathscr{F}_{t}\right] & =\boldsymbol{E}\left\{\Phi[u(z(T))-u(z(T \wedge t))] / / \mathscr{F}_{t}\right\} \\
& =\boldsymbol{E}\left\{\Phi I[T \geqq t] / / \mathscr{F}_{t}\right\}+\Phi(0) I[T<t] .
\end{aligned}
$$

Let us then define $G$ a function on $\Omega$, the space of all paths, by setting:

$$
G(\omega)=\Phi\left[u\left(\omega_{T(\omega)}\right)-u\left(\omega_{0}\right)\right] .
$$

It is then clear by (2.1.2) that on the set $[T \geqq t]$ we have

$$
G\left[\theta_{t}(\omega)\right]=\Phi[u(z(T))-u(z(T \wedge t))]
$$


almost everywhere w.r.t. $\boldsymbol{P}_{0}=\boldsymbol{P}$. We conclude therefore that:

$$
E\left[\Phi / / \mathscr{F}_{t}\right] \leqq \max \left\{\Phi(0), E_{0}\left[G\left[\theta_{t}(\omega)\right] / / \mathscr{F}_{t}\right]\right\} .
$$

By the Markov property (2.1.1) we have on the other hand:

$$
\sup _{t}\left\|\boldsymbol{E}_{0}\left\{G\left[\theta_{t}(\boldsymbol{\omega})\right] / / \mathscr{F}_{t}\right\}\right\|_{\infty}=\sup _{a \in \boldsymbol{C}}\left|\boldsymbol{E}_{a}(G)\right| \text {. }
$$

Observe finally that

$$
\begin{gathered}
\boldsymbol{E}_{a}(G)=P_{a} \Phi[u(\theta)-u(a)] ; \quad a \in \grave{D} \\
\boldsymbol{E}_{a}(G)=\Phi(0) ; \quad a \in \mathcal{G} \stackrel{D}{ }
\end{gathered}
$$

(cf. [6], §2.19). This completes the proof of (2.1.7).

Let us now define another linear operator (which was first considered by B. Mauray in a recent article (cf. [8])).

$$
\begin{gathered}
N: L^{1}(\Omega ; \boldsymbol{P}) \longrightarrow L^{1}(\boldsymbol{T}) \\
N F=f ; \quad f(\theta)=\boldsymbol{E}\left[F / / \boldsymbol{z}_{T}=\theta\right]
\end{gathered}
$$

(i.e., conditional expectation w.r.t. the field generated by the r.v. $z_{T}$ ). Clearly $N$ satisfies

$$
\|N F\|_{L^{p}(\boldsymbol{T})} \leqq\|F\|_{L^{p}(\Omega)} ; \quad 1 \leqq p \leqq+\infty .
$$

We also have the following lemma which is due to B. Mauray [8].

LEMMa 2.2. There exists $C>0$ some positive constant such that for all $F \in \mathrm{BMO}(\Omega)$ we have $N F \in \operatorname{BMO}(T)$ and

$$
\|N F\|_{\text {вмо }(T)} \leqq C\|F\|_{\text {вмо }(\Omega)} \text {. }
$$

Proof (B. Mauray). The proof of the lemma is based on the classical Fefferman duality theorem between $H^{1}$ and BMO. Indeed we have:

$$
\|f\|_{\text {вмо }} \leqq C \sup _{\varphi}\left|\int_{0}^{2 \pi} f \varphi d \theta\right| ; \quad \forall f \in L^{\infty}(\boldsymbol{T})
$$

where the supermum is taken over all $\varphi \in C(T)$ that satisfy

$$
\left\|\sup _{r} u\left(r e^{i \theta}\right)\right\|_{L^{1}(\boldsymbol{T}\rangle} \leqq 1
$$

where $u$ is the harmonic extension of $\varphi$ in the unit disc, and $C$ is a numerical constant.

We also have:

$$
\begin{aligned}
& E(F \cdot G) \leqq C\left\|\sup _{t} F_{t}\right\|_{L^{1}(\Omega)}\|G\|_{\text {вмо }} ; \\
& \forall F \in L^{2}(\Omega), \quad G \in \operatorname{BMO}(\Omega)
\end{aligned}
$$


where $C$ is again a numerical constant. The above two inequalities are the essence of the duality theorem in its classical and probabilistic variant (respectively) (cf. [3]; [9], VI, p. 132). "Let now $F \in L^{\infty}(\Omega)$. By (2.1.8) we have therefore:

$$
\|N F\|_{\text {вмо }(T)} \leqq C \sup _{\varphi}\left|\frac{1}{2 \pi} \int_{0}^{2 \pi} N F \varphi d \theta\right|
$$

where $\varphi$ runs through the same set as in (2.1.8). But for a fixed $\varphi$ in that set we have [cf. (2.1.9)]:

$$
\left|\frac{1}{2 \pi} \int_{0}^{2 \pi} N F \varphi d \theta\right|=|\boldsymbol{E}(F \cdot M \varphi)| \leqq C\|F\|_{\text {вмо }(\Omega)}\left\|\sup _{t}(M \varphi)_{t}\right\|_{L^{1}(\Omega)} .
$$

But from (2.1.4) we also have:

$$
\left\|\sup _{t}(M \varphi)_{t}\right\|_{L^{1}(\Omega)} \leqq C
$$

for some numerical constant. Putting together (2.1.10), (2.1.11) and (2.1.12) we have a proof of the lemma. The two operators $M$ and $N$ are related by:

$$
N M f=f ; \quad \forall f \in L^{1}(\boldsymbol{T}) .
$$

Indeed the above is obvious if $f \in \boldsymbol{C}(\boldsymbol{T})$ and continuity does the rest.

2.3. Proof of Theorem G.J. Let $f \in \operatorname{BMO}(\boldsymbol{T})$ and let us suppose, as we may, that $f$ is real. Let us also suppose that for some $\alpha>0$

$$
\sup _{I} \frac{1}{|I|} \int_{I} e^{\alpha\left|f-f_{I}\right|} d \theta<+\infty
$$

where $I$ runs through all the intervals of $\boldsymbol{T}$. Let us then denote by $X=M f$. It then follows from (0.3.3) and from (2.1.6) that

$$
\sup _{t}\left\|\boldsymbol{E}\left[e^{\beta\left|X-X_{t}\right|} / \mid \mathscr{F}_{t}\right]\right\|_{\infty}<+\infty ; \quad \forall \beta<\alpha .
$$

Applying Theorem G.J. Prob. to $X$ then we obtain

$$
X=Y+Z ; \quad Y \in L^{\infty} ; \quad\|Z\|_{\text {вмо }} \leqq \frac{10}{\alpha} .
$$

But then by Lemma 2.2 and (2.1.13) we have:

$$
f=N X=N Y+N Z=\varphi+\psi ; \quad \varphi=N Y, \quad \psi=N Z
$$

and

$$
\|\varphi\|_{\infty} \leqq\|Y\|_{\infty} ; \quad\|\psi\|_{\text {вмо }(x)} \leqq \frac{10 C}{\alpha}
$$


This proves the nontrivial inequality of Theorem G.J. and completes the proof.

AdDendum I. I am indebted to $R$. Durrett and to the referee (who, incidentally may well be one and the same person) for the following drastic simplification of Lemma 1.2.

Let us adopt the notations and the hypothesis of Lemma 1.2 imposing however the additional condition that the sequence $\left\{T_{i} ; i \geqq 0\right\}$ is $\gamma$-Graded (with $0<\gamma<1$ arbitrary). For the use that we will make of the lemma this is good enough. Let us define

$$
n_{1}(\omega)=\inf \left\{i \geqq 1: \omega \in A_{i}\right\}
$$

and continue

$$
n_{j+1}(\omega)=\inf \left\{i>n_{j}(\omega) ; \omega \in A_{i}\right\} .
$$

With the above notation it is then clear that the $\left\{S_{j}, j \geqq 0\right\}$ that were defined in the proof of Lemma 1.2 can also be defined by

$$
S_{j}=T_{n_{j}(\omega)} ; \quad j=1,2, \cdots
$$

(with the convention $T_{\infty}=\infty$ ), and that therefore:

$$
F=\sum_{j=1}^{\infty} I\left[S_{j}<+\infty\right] \text {. }
$$

So far, of course, this is but a notational improvement; what is nice however is that under the above additional condition and with this new notation it is almost evident that the $\left\{S_{j} ; j \geqq 1\right\}$ are $\gamma$-Graded.

Indeed one can prove very easily that

$$
\left[n_{j}=k\right] \in \mathscr{F}_{T_{k}} ; \quad\left[n_{j}=k\right] \in \mathscr{F}_{S_{j}}
$$

and conclude from this that

$$
\begin{aligned}
E\left[S_{j+1}<\right. & \left.+\infty / / \mathscr{F}_{s j}\right] I\left[n_{j}=k\right] \\
& =E\left[n_{j+1}<+\infty, T_{n_{j+1}}<+\infty / / \mathscr{F}_{T_{k}}\right] I\left[n_{j}=k\right] \\
& \leqq E\left[T_{k+1}<+\infty / / \mathscr{F}_{T_{k}}\right] I\left[n_{j}=k\right] \leqq \gamma
\end{aligned}
$$

which concludes the proof of the lemma.

ADDENDUM II. I am indebted to the referee and to $\mathrm{P}$. Jones for the following.

REMARK. To deduce the Garnett-Jones theorem for $\boldsymbol{R}^{n}(n \geqq 2)$ one can use the conformal mapping that mape $\boldsymbol{R}^{n}$ onto $\Sigma_{n}$ the unit sphere. This mapping preserves BMO. To obtain the theorem for 
$\operatorname{BMO}\left(\Sigma_{n}\right)$ one then has to consider harmonic functions in the unit ball and Brownian motion starting from its center which is much simpler then starting Brownian motion "at $\infty$ in $\boldsymbol{R}_{+}^{n+1}$ ".

ADDENDUM III. The proof of Theorem 1.2 as given above can easily be adapted to yield the following more precise result:

THEOREM 1.2'. Let $F$ be a real valued function in $\mathrm{BMO}(\Omega)$ and let $\alpha^{+}, \alpha^{-}>0$ be two positive constants that satisfy

$$
\sup _{t}\left\{\boldsymbol{E}\left(e^{\alpha^{+}\left(F-F_{t}\right)} / / \mathscr{F}_{t}\right), \boldsymbol{E}\left(e^{-\alpha^{-}\left(F-F^{t}\right)} / / \mathscr{F}_{t}\right)\right\} \leqq K \quad \text { a.e. } \omega \in \Omega
$$

where $K$ is a positive constant. Then for every $\lambda \geqq \lambda_{0}\left[\lambda_{0}\right.$ depends on $\alpha^{+}, \alpha^{-}$and $K$ ] there exists a decomposition of $F$

$$
F=\lambda \Phi^{+}-\lambda \Phi^{-}+\Psi+\boldsymbol{E}(\boldsymbol{F})
$$

where $\|\Psi\|_{\infty} \leqq \lambda$ and where the two functions $\Phi^{+}$and $\Phi^{-}$are $\gamma^{+}$and $\gamma^{-}$-Graded (respectively) with

$$
\gamma^{+}=K e^{\alpha^{+} \lambda}\left(1-K e^{\alpha^{+\lambda}}\right)^{-1} ; \quad \gamma^{-}=K e^{\alpha^{-\lambda}}\left(1-K e^{\alpha^{-\lambda}}\right)^{-1} .
$$

The reader can supply the details, I am sure. Observe however that for the proof of Theorem 1.2' the full thrust of Lemma 1.2, as given in the text, seems to be needed, and the simplification given in Addendum I no longer seems to work.

Let now $W=e^{f}$ be a positive function on $\Omega$ (with $f \in L^{1}(\Omega)$ ). We then say that $W \in A_{p}$ for some $p \in(1,+\infty)$ if

$$
\sup _{t}\left\{\left\|\boldsymbol{E}\left(e^{f-f_{t}} / / \mathscr{F}_{t}\right)\right\|_{\infty},\left\|\boldsymbol{E}\left(e^{-(1 /(p-1))\left(f-f_{t}\right)} / / \mathscr{F}_{t}\right)\right\|_{\infty}\right\} \leqq \infty .
$$

We also say that $f \in A_{1}$ if there exists some $K>0$ s.t.

$$
\begin{aligned}
& \boldsymbol{E}\left(e^{f-f_{t}} / / \mathscr{F}_{t}\right) \leqq K \quad \text { a.e. } \quad \omega \in \Omega \\
& f-f_{t} \geqq-K \quad \text { a.e. } \quad \omega \in \Omega
\end{aligned}
$$

for all $t \geqq 0$.

Theorem $1.2^{\prime}$ and the well known fact that for all $W \in A_{p}$ (some $1<p<+\infty)$ there exists some $\varepsilon>0$ s.t. $W^{1+\varepsilon} \in A_{p}$ (cf. [12], [13]) imply the following probabilistic version of $\mathrm{P}$. Jones' factorization theorem [13].

Theorem (P. Jones Prob.). A weight $W$ satisfies the $A_{p}$ condition, $1<p<\infty$ if and only if

$$
W=W_{1}\left(W_{2}\right)^{1-p}
$$

where $W_{1}, W_{2} \in A_{1}$. 
The interested reader should look at [13] for the significance of the above theorem.

\section{REFERENCES}

1. R. M. Blumenthal and R. K. Getoor, Markov Processes and Potential Theory, Academmic Press, 1968.

2. D. L. Burkholder, R. F. Gundy, and M. L. Silverstein, A maximal function character. isation of the class $H^{p}$, Trans. Amer. Math. Soc., 157 (1971), 137-153.

3. C. Fefferman and E. M. Stein, $H^{p}$ spaces of several variables, Acta Math., 129 (1972), 137-193.

4. J. B. Garnett and P. W. Jones, The distance in BMO to $L^{\infty}$, Ann. Math., 108 (1978), 373-393.

5. K. Itô, Multiple Wiener integrals, J. Math. Soc. Japan, 3 (1951), 157-169.

6. - Stochastic Processes, Lecture Notes Series No. 16, Aarhus Universitet, 1968/ 69.

7. F. John and L. Nirenberg, On functions of bounded mean oscillation, Comm. Pure Appl. Math., 14 (1961), 415-426.

8. B. Mauray, C. R. Acad. Sciences, Paris, 1978, t. 288, Série A, pp. 271-273 and 865867.

9. P. A. Meyer, Seminaire de Probabilités $X$ (Resp. XI), Springer Verlag: Lecture Notes in Mathematics No. 511 (resp. No. 581).

10. J. Neveu, Bases Mathematiques du Calcul des Probabilités, Masson et Cie, Paris, 1964. 11. E. M. Stein, Singular Integrals and Differentiability Properties of Functions, Princeton University Press, 1970.

12. R. R. Coifman, and C. Fefferman, Weighted norm inequalities for maximal functions and singular integrals, Studia Math., 51 (1974), 241-250.

13. Peter W. Jones, Factorization of $A_{p}$ weights, to appear in Ann. Math.

Received June 15, 1979.

Universite Paris-Sud, Orsay, France (91)

AND

University of California, Los Angeles 



\section{PACIFIC JOURNAL OF MATHEMATICS}

\section{EDITORS}

DONALD BABBITT (Managing Editor)

University of Galifornia

Los Angeles, California 90024

HUGO RossI

University of Utah

Salt Lake City, UT 84112

C. C. MOORE AND ANDREW OGG

University of California

Berkeley, CA 94720
J. DUGUNDJI

Department of Mathematics University of Southern California Los Angeles, California 90007

R. FINN AND J. MILGRAM Stanford University

Stanford, California 94305

ASSOCIATE EDITORS

E. F. BECKENBACH

B. H. NeumanN

F. WOLF

K. YosHidA

\section{SUPPORTING INSTITUTIONS}

UNIVERSITY OF BRITISH COLUMBIA UNIVERSITY OF SOUTHERN CALIFONIA CALIFORNIA INSTITUTE OF TECHNOLOGY UNIVERSITY OF CALIFORNIA MONTANA STATE UNIVERSITY STANFORD UNIVERSITY UNIVERSITY OF HAWAII UNIVERSITY OF NEVADA, RENO UNIVERSITY OF TOKYO U'NIVERSITY OF UTAH NEW MEXICO STATE UNIVERSITY WASHINGTON STATE UNIVERSITY OREGON STATE UNIVERSITY UNIVERSITY OF OREGON UNIVERSITY OF WASHINGTON 


\section{Pacific Journal of Mathematics}

\section{Vol. 90, No. $1 \quad$ September, 1980}

Shashi Prabha Arya and M. K. Singal, On the locally countable sum

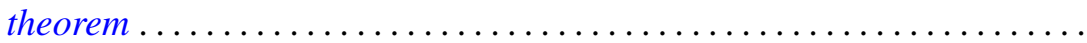

John Theodore Baldwin and David William Kueker, Ramsey quantifiers and

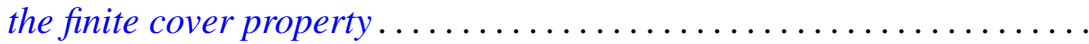

Richard Body and Roy Rene Douglas, Unique factorization of rational

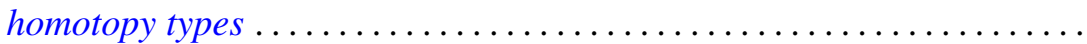

Ethan Bolker and Ben G. Roth, When is a bipartite graph a rigid

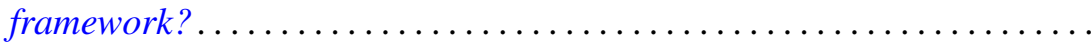

Alicia B. Winslow, Continua in the Stone-Čech remainder of $R^{2} \ldots \ldots \ldots$

Richard D. Carmichael and Elmer Kinji Hayashi, Analytic functions in tubes which are representable by Fourier-Laplace integrals ..............

Stephen D. Cohen, The Galois group of a polynomial with two indeterminate coefficients ..............................

Russell Allan Johnson, Strong liftings commuting with minimal distal

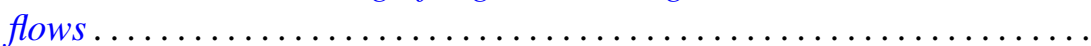

Elgin Harold Johnston, The boundary modulus of continuity of harmonic

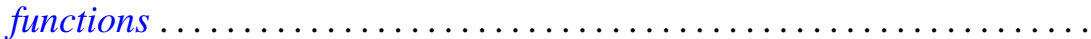

Akio Kawauchi and Takao Matumoto, An estimate of infinite cyclic

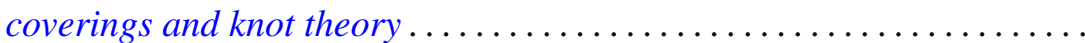

Keith Milo Kendig, Moiré phenomena in algebraic geometry: rational alternations in $\mathbf{R}^{2}$...

Roger T. Lewis and Lynne C. Wright, Comparison and oscillation criteria for selfadjoint vector-matrix differential equations .

Teck Cheong Lim, Asymptotic centers and nonexpansive mappings in conjugate Banach spaces .......................

David John Lutzer and Robert Allen McCoy, Category in function spaces.

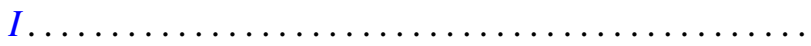

Richard A. Mollin, Induced p-elements in the Schur group ...

Jonathan Simon, Wirtinger approximations and the knot groups of $F^{n}$ in $S^{n+2}$

Robert L. Snider, The zero divisor conjecture for some solvable groups...

H. M. (Hari Mohan) Srivastava, A note on the Konhauser sets of biorthogonal polynomials suggested by the Laguerre polynomials...

Nicholas Th. Varopoulos, A probabilistic proof of the Garnett-Jones theorem on BMO.

Frank Arvey Wattenberg, $[0, \infty]$-valued, translation invariant measures on $N$ and the Dedekind completion of ${ }^{*} R \ldots \ldots \ldots \ldots . .$. 